\title{
A Paradigm Shift in Low Dose Radiation Biology
}

\author{
Z. Alatas ${ }^{*}$ \\ Center for Radiation Safety Technology and Metrology, National Nuclear Energy Agency, \\ Jl. Lebak Bulus Raya No.49, PO Box 7043 JKSKL, Jakarta Selatan 12070, Indonesia
}

\section{ARTICLE INFO}

Article history:

Received 14 October 2014

Received in revised form 1 April 2015

Accepted 14 April 2015

\section{Keywords:}

radiobiology paradigm

low dose radiation

genomic instability

bystander effect

adaptive response

\begin{abstract}
A B S T R A C T
When ionizing radiation traverses biological material, some energy depositions occur and ionize directly deoxyribonucleic acid (DNA) molecules, the critical target. A classical paradigm in radiobiology is that the deposition of energy in the cell nucleus and the resulting damage to DNA are responsible for the detrimental biological effects of radiation. It is presumed that no radiation effect would be expected in cells that receive no direct radiation exposure through nucleus. The risks of exposure to low dose ionizing radiation are estimated by extrapolating from data obtained after exposure to high dose radiation. However, the validity of using this dose-response model is controversial because evidence accumulated over the past decade has indicated that living organisms, including humans, respond differently to low dose radiation than they do to high dose radiation. Moreover, recent experimental evidences from many laboratories reveal the fact that radiation effects also occur in cells that were not exposed to radiation and in the progeny of irradiated cells at delayed times after radiation exposure where cells do not encounter direct DNA damage. Recently, the classical paradigm in radiobiology has been shifted from the nucleus, specifically the DNA, as the principal target for the biological effects of radiation to cells. The universality of target theory has been challenged by phenomena of radiation-induced genomic instability, bystander effect and adaptive response. The new radiation biology paradigm would cover both targeted and non-targeted effects of ionizing radiation. The mechanisms underlying these responses involve biochemical/molecular signals that respond to targeted and non-targeted events. These results brought in understanding that the biological response to low dose radiation at tissue or organism level is a complex process of integrated response of cellular targets as well as extra-cellular factors. Biological understanding of the effects of radiation can be used to improve the assessment of low dose radiation risk. In this article, the mechanisms of targeted and non-targeted responses, and interrelation between the phenomena on cellular injury after exposure to low doses of radiation as they relate to low dose radiation effects will be reviewed.
\end{abstract}

(C) 2015 Atom Indonesia. All rights reserved

\section{INTRODUCTION}

Radiation is potentially harmful to humans because the produced ionization can significantly alter the structure of molecules within a living cell. For many years, the central dogma in radiobiology following exposure to all kinds of

\footnotetext{
*Corresponding author.

E-mail address: zalatas@batan.go.id

DOI: http://dx.doi.org/10.17146/aij.2015.379
}

ionizing radiation has been that the nucleus, especially deoxyribonucleic acid (DNA), is the principal target for the biological effects of radiation. The deleterious effects of ionizing radiation include mutation induction, chromosomal rearrangement, cellular transformation, carcinogenesis and cell death [1]. Extrapolation of data from epidemiological investigations requires knowledge of the mechanisms of radiation action and post-irradiation processes that specifically relate to health effects. Biological understanding of the 
effects of radiation can be used to improve the assessment of low dose radiation risk. The health effects of concern following low dose radiation exposure are cancer and hereditary effects. The lack of understanding of the mechanisms of action of radiation at low doses is a major contributor to the current uncertainty on low dose risk estimates. For the purposes of estimating of risks to human health from exposure to low doses of radiation that may be received by the general public, workers and patients undergoing medical procedure, United Nations Scientific Committee on the Effects of Atomic Radiation (UNSCEAR) has now defined low doses as those of $100 \mathrm{mSv}$ or less [2]. This newly agreed definition is consistent with that used by the Biological Effects of Ionizing Radiation (BEIR) VII Report [3] and International Commission of Radiological Protection (ICRP) [4].

A classic paradigm in radiation biology is that all radiation effects on cells, tissues and organisms are due to the direct action of radiation on living tissue. Based on this, possible risks from exposure to low dose ionizing radiation are estimated by extrapolating from data obtained after exposure to higher doses of radiation, using a linear non-threshold relationship between dose and subsequent effect. However, the validity of using this dose-response relationship is controversial because evidence accumulated over the past decade has indicated that living organisms, including humans, respond differently to low dose radiation than they do to high dose radiation [2,3].

There is shift in thinking from targeted or classical theory that a radiation must hit DNA as the only target in order to cause cell damage (effector), to the notion that a radiation can hit more than one target in exhibition of biological effect, which can be the same cell and/or other cells (multiple effectors). Meaning that cellular damage is expressed in unirradiated neighboring cells near to an irradiated cell or cells and in the progeny of irradiated cells at delayed times after radiation exposure. These results brought in a paradigm shift in understanding that the biological response to radiation at tissue or organism level is a complex process of integrated response of cellular targets as well as extra-cellular factors. Phenomena associated with fundamental shift in paradigms from deterministic hit-effect relationships to complicated ongoing cellular responses are bystander effects, genomic instability, and adaptive responses that all three have been specifically observed in response to low dose radiation and a long time after the initial radiation exposure [3-8].

The application of the new techniques and knowledge to radiobiology has started to provide some new insights into the mechanisms of low dose radiation action. This document will provide a brief summary of conventional mechanisms of radiation action on targeted effects, the major developments of non-targeted effects occurring after exposure to low doses of radiation and interaction between the phenomena.

\section{EXPERIMENTAL METHODS}

\section{Interaction of radiation with biological material}

The biological effects of ionizing radiation are primarily due to its ability to ionize the material in the cells, which make up the body's tissues. By this mechanism energy is transferred from the radiation to the material. Radiation dose is the amount of energy per unit of biological material. Linear Energy Transfer (LET) is a physical parameter to describe average energy released per unit length of the track in traversed material. Radiations such as neutrons and alpha particles that cause dense ionization along their track are called high LET radiation. Low LET radiations such as $\mathrm{X}$-ray and gamma radiation produce ionizations sparsely along their track and almost homogeneously within a cell. At the same dose, the low LET radiations induce the same number of radicals more sparsely within a cell, whereas the high LET radiations transfer most of their energy to a small region of the cell, therefore, high LET radiations are more destructive to biological material than low LET radiations. The localized DNA damage caused by dense ionizations from high LET radiations is more difficult to repair than the diffuse DNA damage caused by the sparse ionizations from low LET radiations [1,9].

Radiation induced ionizations may act directly on the cellular component molecules or through the indirect action of free radicals which are formed through radiation energy deposition in water molecules in cells or tissues. Radicals react with nearby molecules in a very short time resulting in breakage of chemical bonds or oxidation of the affected molecules. The traditional thinking has been that the biological effects of ionizing radiation occur in irradiated cells as a consequence of the DNA damage. This implies that biological effects occur only in irradiated cells, radiation traversal through the nucleus of the cell is a prerequisite to produce a biological response, and DNA is the target molecule in the cell. Three possible actions will occur when ionizing radiation sufficient to cause cellular damage i.e. (1) if the damage is too 
severe, the cell may die; (2) if the cell is not severely damaged, it might be able to repair itself and continue functioning, but could lose its ability to divide; or (3) a damaged normal cell might mutate, which may cause cancer or genetic effects $[9,10]$.

Low doses of radiation would tend to damage at the level of the cell or alter the genetic code (DNA) of irradiated cells, and thus the changes may not be observed for many years after exposure. The genes mainly involved in this cellular response to radiation are those involved in cell-cycle regulation, signal transduction, intercellular signaling, development and DNA damage repair. The mutation of genes result in alteration of their expression, loss of gene products (proteins) or change in protein properties or amounts that may disrupted the biochemical balance of the cell $[1,11]$.

\section{RESULTS AND DISCUSSION}

\section{Health effects of low dose radiation}

The primary health effects of low dose radiation are hereditary effects and the development of cancer. These diseases can affect a range of different organs/tissues in the body and arise naturally that be relatively common in the general population. The information obtained from direct observation of excess disease in an irradiated population through epidemiological studies and indirect observation in animals and cultured cells as the experimental models of disease.

Hereditary effects are those effects observed in the off spring of parent(s) that has or have been irradiated prior to conception as the result of a mutation produced in the reproductive cells. Depending on whether the altered genes are dominant or recessive, these effects may appear in the exposed person's direct offspring, or may appear several generations later. Studies with experimental animals clearly demonstrate that radiation can cause hereditary effects whereas no evidence for the induction of hereditary effects by radiation in humans has been obtained [1-3].

Cancer is a general term used to describe major disturbances in the growth pattern of primitive, so called stem cells in body organs. These primitive stem cells normally develop and divide in a coordinated way to form the specialist cells of the organ but abnormal growth and arrested development can lead to a mass of cells in a given organ which is termed a solid tumor. There is strong epidemiological evidence that radiation exposure of humans can lead to excess solid tumors in many body organs and leukemia in the blood system.
There is also growing information on the cellular/molecular mechanisms through which these cancers can arise. Human cancer arise from the accumulation of multiple genetic abnormalities which must occur in critical genes that regulate proliferation and differentiation. Cancer induced by radiation has long latent periods such as 10 years for leukemia and over 30 years for solid cancer [1-3].

Research and biological knowledge of molecular and cellular mechanisms confirm that carcinogenesis is a highly complex multi-step process. Generally carcinogenesis starts by mutation of one or more genes of the DNA of a single somatic cell in a body organ. This implies that mutations induced by radiation due to gene mutation and/or chromosomal damage that can be detected within 24 hours of radiation exposure are not directly responsible for initiating carcinogenesis in normal human cells. Subsequent cancer development and the onset of malignancy are assured to proceed in a multistep model which each step has been associated with mutation or other changes involving cellular genes. However, such mutations induce genetic instability that make cells more sensitive to accumulation of additional genetic abnormalities caused by exposure to additional radiation doses, chemical mutagens and carcinogens, tumor promoters, oncogenic viruses, or their combinations. Cells may continue to carry genetic abnormalities for a long time until the expression of genes regulating differentiation is altered. No universal pattern of response has been identified and not all genes change in expression level following radiation exposure [2,3,10,11].

Radiation is well known to play role mainly in inducing initiating mutations in proto-oncogenes or tumour suppressor genes that have normal cellular functions in cell growth, development and regulation. Cancer initiation involves a loss of regulation of growth, reproduction and development in somatic stem cells, i.e the loss of control over the cell reproduction cycle and differentiation processes. Point mutations and chromosomal damage play roles in the initiation of neoplasia. Initiation can result from the inactivation of tumour suppressor genes, some of which play a central role in the control of the cell cycle. Although cells may have undergone initiating changes, they will not express their properties until they are stimulated or promoted to reproduce. The promoting agents may be independent of the initiation agent $[2,12]$. The growth of cancer cells may also be regulated to some extent by the tissue microenvironment affected by radiation involving can reactive oxygen, inflammation and transforming growth factor $\beta$ (TGF $\beta$ ) signaling [13]. The main drivers of low 
dose radiation health effects are the direct induction of targeted mutations in DNA, the contribution of non-targeted and delayed effects distant from radiation-induced DNA lesions.

The induction and development of cancer after radiation is not simply a matter of the stepwise accumulation of mutations in the DNA of the relevant cells. Moreover, the adaptation of cells and tissues to low dose radiation might cause them to become more resistant to cancer development. The radiation effects of radiation on the immune systems that recognize or destroy abnormal cells could influence the possibility of cancer development.

\section{Targeted effects of low dose ionizing radiation}

When ionizing radiation traverses biological material, some energy depositions occur directly in biologically important molecules causing ionizations. The radiation damage to biological systems were explained based on target theory of radiation which suggests that death of a cell after radiation exposure is caused by the inactivation of specific targets within cells. Cells contain at least one target as the critical site that must be hit by radiation so as to kill or change a cell. Therefore, radiation damage outside of the target should not cause cell death. It is widely accepted that nuclear DNA is the critical target for detrimental biological effects of radiation. The theory of DNA critical target not only could explain mechanisms in radiation biology but also accelerate the progress of cellular and molecular radiation biology [9].

\section{DNA as the target of radiation}

It has long been a central radiobiological dogma that the biological effects of ionizing radiation are the results of the direct ionization of cell structures, particularly DNA, or indirect ionization via free radicals as the water radiolysis products. The main subcellular target for radiationassociated cellular alteration are the DNA molecules in the nucleus. Damage to DNA molecules residing in the chromosomes is the main initiating event of radiation that causes long-term harm to organs and tissues of the body. The DNA codes for about 30,000 genes that coordinate all functions in each cell that can be affected at low doses of radiation. Radiation is able to simultaneously damage both strands of DNA resulting in complex chemical changes. Reaction between free radicals as the results of water radiolysis, especially hydroxyl radical with DNA will produces damages on both the sugar and the bases of DNA that leads into single DNA lesions such as base damage (BD), abasic sites (AS), single strand breaks (SSBs), DNA-protein cross links (DPC), or double strand breaks (DSBs) as well as clustered DNA lesions (locally multiply damaged sites, LMDS). The latter might involve one or more DSB, several SSB as well as BD. Therefore, LMDS may be more difficult to repair or fail to repair $[2,10]$.

Cells have complex signal transduction, cell cycle checkpoint and repair pathways to respond to the DNA damage and to restore the genetic integrity of a cell. BD, AS and SSBs are repaired by different processes like base excision repair, nucleotide excision repair (NER), and single strand break repair. DPC are repaired by NER and homologous recombination repair (HRR). DSBs are the critical lesions that can lead to cell death via the formation of lethal chromosomal aberrations or the induction of apoptosis if not repaired appropriately. To protect cells from the potentially deleterious effects, there are two principal DSBs repair mechanisms i.e. HRR and non homologous end joining (NHEJ) which act at different phase of the cell cycle. Briefly, DSB repair by $\mathrm{HR}$ requires an undamaged template molecule that contains a homologous DNA sequence, typically on the sister chromatid in the $\mathrm{S}$ and G2 phases of the cell cycle. In contrast, NHEJ which may occur in all cell-cycle phases, does not require an undamaged partner and does not rely on extensive homologies between the recombining ends [14-16].

DSBs and LMDS are likely to be of greatest importance in terms of causing lasting mutations. Even the lowest doses of radiation may induce DNA damage that may be converted into DNA sequence mutations since none of the DNA repair systems is completely error free. Chromosome aberrations that arise from unrepaired or inaccurately repaired DSB represent a readily visible type of DNA damage caused by ionizing radiation that are observable during mitosis using microscope. Chromosome aberrations commonly induced by radiation are dicentrics, rings, acentric fragments, deletions, translocations, and anaphase bridges. The frequency of aberrations is radiation dose dependent and can be used as an indication of dose received under some conditions. The range of DNA damages lead to cellular endpoints such as chromosome aberrations, mutagenesis, and genomic instability which are contributing to malignant cell transformation. Damage to other cellular components (epigenetic changes) may influence the functioning of the cell and progression to the malignant state [1-3]. 
Importantly, the key mutational events in cancer development are frequently dependent on the organ in which the irradiated cell is located and fall into two general categories, small specific mutations in single genes and mutations involving loss of DNA._Cells have a number of biochemical pathways capable of recognizing and dealing with specific forms of damage. One gene that plays a key role is the tumour suppressor TP53, which is lost or mutated in more than half of all human tumours. The p53 protein produced by the gene controls both arrest of the cell cycle and one pathway of apoptosis (the programmed cell death that is instrumental in preventing some damaged cells from progressing to the transformed, malignant growth stage). Some such biochemical pathways are also implicated in stress response or adaptation processes that act to limit the extent or outcome of damage $[1,2]$.

A series of several mutations (perhaps two to seven) in the cell genetic code are usually required to result in a fully transformed cell capable of leading to a cancer. In spontaneous cancers, these mutations will have occurred randomly during life. Thus, even after initial cell transformation and promotion, further mutations are needed to complete the clonal transition from preneoplasia to cancer. The whole process is called multi-stage carcinogenesis $[2,3,10]$. Based on the classical radiation carcinogenesis, ionizing radiation damages primarily nuclear DNA inducing targeted DNA mutations in the unrepaired stem cells correctly thus initiating the cancer development process. Secondary mutations accumulate leading to development of a malignant neoplasm.

\section{Non-targeted effects of ionizing radiation}

The classic descriptions of radiation-induced damage in cells, as just discussed, are based on responses triggered by DNA damage in the cells exposed to radiation. In the past 15 years, there has been increasing acceptance that so-called nontargeted effects can also occur in which biological responses that are not directly related to the amount of energy deposited in the DNA of the cells traversed by the radiation, but can result from nonDNA-damage-initiated events and can be seen in non-irradiated cells. An essential feature of nontargeted effects is the effect that do not require a direct nuclear exposure to radiation to be expressed and are particularly significant at low doses radiation. The effects considered to be non-targeted effects of radiation are genomic instability, bystander effects, and adaptive responses. These three effects involve intracellular signaling or communication that may have in common involvement of oxidative stress, inflammatory-like response pathways, and induction of cytokines/growth factors. The interactions and signaling induced are quite complex, for example, radiation can activate cell-mediated inflammatory processes that cause DNA damage in stem cells $[17,18]$ or radiation-induced signals from fibroblasts can induce apoptosis in transformed epithelial cells [19]. Although these effects have generally been considered in terms of relevance to carcinogenesis, late chronic side effects in irradiated normal tissues may be associated with similar oxidative and inflammatory effects $[18,20]$ suggesting these phenomena could may play roles in the mechanisms of normal tissue damage.

Genomic instability is the increase in chromosomal instability, mutations, apoptosis, or other deleterious effects in the progeny of irradiated cells, often many generations after the exposure [17,21,22]. The high frequency of genomic instability in progeny of irradiated cells of normal tissue origin is not consistent with a mutational mechanism. Bystander effects are cellular responses occurring in non irradiated cells that are near to or sharing medium with irradiated cells [21,23]. The responses include increased reactive oxygen species (ROS), DNA damage, mutagenesis, changes in gene expression and decreased clonogenic survival in bystander cells that can be seen after irradiation of a single cell in a population of hundreds of cells [21,23]. On the other hand, adaptive response is a phenomenon whereby the detrimental effects of DNA-damaging agents can be mitigated if the cells are exposed to a prior stress and low-dose ionizing radiation was first identified to have an effect [24]. This radioadaptive response is the acquirement of cellular resistance to the genotoxic effects of radiation by prior exposure to low-dose radiation. The potential relevance of these non-targeted effects to normal tissue radiation biology will be described briefly below.

\section{Radiation-induced genomic instability}

Induction of genomic instability can be described as if a single cell is irradiated and survives, it may produce daughter cells that over several generation have increasing numbers of alterations in their genomes, even though the daughter cells themselves were not irradiated. This phenomenon that surprisingly frequent event in the progeny of surviving cells can be defined as the persistent production of genomic changes in the progeny of surviving cells after irradiation, which 
could be detected as increased mutation rates on genes and DNA sequences and delayed chromosomal aberrations and reproductive cell death. The survivors of irradiated cells showed different genetic lesions than initially occurred in the radiation exposed parental cells indicating that the target for induced instability is large [2].

There appear to be multiple pathways for initiating and perpetuating radiation-induced genomic instability and the relative contributions of different pathways may depend on the genetic background of the target cell or organism. A long term study of low dose rate (20-200 mGy/day) $\gamma$-ray-exposed $\mathrm{C} 3 \mathrm{H}$ mice identified a contribution of indirect effects of radiation in the induction of complex chromosomal aberrations in spleen cells [25]. In utero irradiation of BALB/c mice has been observed to lead to a persistent elevation in mutation frequency at expanded simple tandem repeat (ESTR) loci in somatic tissues which can pass transgenerationally to an F1 generation [26]. Elevated mutation rates at an ESTR locus and at a protein-coding gene (hprt), possibly due to the presence of persistent DNA damage, were also observed in the first generation offspring of irradiated male mice [27]. The three studies above provide evidence for the induction of transmissible genomic instability by radiation in mice.

The nature of directly induced damage and a reduced ability to repair DNA damage may promote instability. The process of genomic instability not only induced in progeny of directly irradiated cells but may also be induced in bystander cells. A study provides evidence for non direct induction of transmissible genomic instability. The authors ascribe this induction to factors released from macrophages including tumour necrosis factor- $\alpha$, nitric oxide and superoxide [18]. It seems likely that there are multiple transmissible instabilities that require improved functional definition and mechanistic understanding before their importance in radiation-induced health effects can be properly assessed.

\section{Radiation-induced bystander effect}

Radiation-induced bystander effect is defined as the induction of biological effects in cells that are not directly traversed by radiation or a charged particle, but are neighbors of cells that were irradiated. These cells might have been in the same radiation environment or they might be nonirradiated cells that received culture medium from irradiated cells. The phenomenon is in terms of chromosomal aberrations, alterations in gene expression and induction of cell lethality. The bystander response can be considered as the strongest evidence in support of non-targeted radiation effects that were observed in in vivo and in in vitro [2].

Studies using a charged particle microbeam provided the first clear-cut indication of a radiationinduced bystander phenomenon. Following a low dose of alpha particles, a larger proportion of cells showed biological damage than was estimated to have been hit by an $\alpha$-particle. To demonstrate the induction of a bystander effect unequivocally, studies were conducted in which a defined proportion of cells in a confluent monolayer that were irradiated individually with a defined number of $\alpha$-particles. Changes in gene expression, mutation induction, delayed lethality, apoptosis and micronuclei formation were also described in cells exposed to very low fluences of a particles [28].

Although the molecular mechanisms underlying the bystander effect are not yet clear, it is evident that intercellular signal transduction between irradiated cells (target cells) and unirradiated cells (bystander cells) could play a major role in multiple mechanisms of bystander responses or communication. Four possible models for intercellular signaling pathways, capable of producing the radiation-induced bystander response that have been proposed are through (a) medium or transmitted via gap junctions intercellular communication; (b) interactions between ligands and their specific receptors; (c) interaction between the secreted factors and their specific receptors; (d) plasma membranes [5,29,30]. Models (c) and (d) assume that irradiated and unirradiated cells are non-adjacent and distant from each other, and that the bystander transmission factors must be soluble elements secreted from the irradiated cells. Evidence from several systems implicates that there are a range of potential mediators of bystander signals secreted by cells after exposed radiation that can stimulate or modify responses in undamaged cells and even kill the cells that were not damaged that have been identified including ROS [29], reactive nitrogen species, nitric oxide [31], the cytokine such as interleukin-8 (IL-8) [32] and TGF $\beta$ [33], oxidative enzymes such as Cox-2 [21], the transcription factor NF-kB [22] and mitochondria [34] and other inflammatory response markers [35]. Several studies with 3D tissue models have also demonstrated bystander responses and these responses can occur over significant distances (e.g., up to $1 \mathrm{~mm}$ ) and for times up to 48 hours [30,36,37].

Recently, radiation-induced bystander responses were also observed in vivo $[23,38,39]$ and 
this can be associated with increased cancer risk. But it is important to note that in addition to ionising radiation, a number of other agents have been reported to induce bystander-type responses suggesting that ionizing radiation-induced bystander effects reflect a general stress response. This may have implications for the significance of bystander effects for low dose radiation risk assessment. The occurrence of radiation-induced bystander responses in vivo would be of relevance to human health.

Not all bystander effects are detrimental to the bystander cell. Bystander effect is a protective mechanism by the function of eliminating potentially damaged cells in the vicinity of radiation induced DNA damage by apoptosis and increased differentiation. Implications of bystander effects for radiation protection could be important and might contribute to better estimation of cancer risk. In particular, bystander effect is potentially significant for radiation protection issues and may have implications for the applicability of the LNT model in extrapolating radiation risk data into the low dose region.

\section{Radiation-induced adaptive response}

Originally the radiation-induced adaptive response was described as the decrease in frequency of chromosome aberrations in irradiated human lymphocytes when the cells were treated with a low priming dose prior to a larger challenge dose [40]. Since then, the observations have been reported in labs with a variety of test systems that has been seen in vitro and in vivo $[22,41]$. Adaptive response to radiation in cells is characterized by a reduction in radiobiological response in cells pretreated with an initial low dose (priming dose) of ionizing radiation activating a repair mechanism that reduces the response to a subsequent challenge larger dose. Apparently, the range of initial or priming doses is limited and the time for deliberating the challenge dose with a reasonable magnitude is critical [2].

The radioadaptive response is defined by a "window" for a priming dose (about $0.01-0.2$ Gy) in culture cells which is the dose required to induce an effective protective signaling mechanism and an "interval period" between a priming and challenge exposure to radiation [3]. The radioadaptive response has an optimum dose range below $0.1 \mathrm{~Gy}$ [43], occurs in metabolically active cells but not in dormant G0 cells [16], represents an immediate early response being expressed maximally at $4-6 \mathrm{~h}$ after irradiation and continues for more than 20 hours [17,42]. Higher doses are not only incapable of inducing adaptation, but also immediately erase the adapted condition [42], indicating an involvement of some feedback regulatory mechanisms. If the priming dose is over $0.2 \mathrm{~Gy}$, adaptive responses are barely induced, and when it is over $0.5 \mathrm{~Gy}$, adaptive responses are almost never induced [43].

There have been numerous reports demonstrating the presence of adaptive response in a variety of mammalian cells observed using low LET radiations and various endpoints such as chromosomal aberrations [3], micronuclei formation [44], mutation induction and spectrum [45], and radiosensitivity [23] neoplastic transformation [46], apoptosis [5], cell proliferation [47], and cell killing [5].

A possible molecular mechanism of adaptive response to low-dose ionizing irradiation has been related to the repair of DSBs and p53 protein played a key role in the adaptive response. The adaptive response to low-dose ionizing irradiation thus, favors the involvement of the NHEJ pathways. The p53 protein channels the radiation-induced DNA DSBs into an adaptive legitimate repair pathway, and hence turn off the signals to an alternative pathway to illegitimate repair and apoptosis [48]. Cytogenetic adaptive response of human lymphoblastoid cells is used to determine the modification of gene expression in adapted cells and to identify the genes that are associated with reduction of radiation effects by a comprehensive assay using cDNA microarray analyses. Whereas genes associated with cellular proliferation, signal transduction, apoptosis, ubiquitin-dependent protein degradation, translation, protein modification and DNA double-strand break repair were downregulated, genes associated with base excision repair, cell cycle control, signal transduction, and stress response were up-regulated [49].

The impact on health of relatively short-lived modification in radiosensitivity is not clear. Radioadaptive response can be seen as a biological defense mechanism in which low-dose ionizing irradiation elicits cellular resistance to the genotoxic effects of subsequent irradiation. Since adaptive response is demonstrated at low doses and doserates, it may someday have an impact on radiation protection standards and regulations and may be useful in radiation therapy to protect normal tissue.

\section{Interrelationships among low dose responses to radiation}

It is unlikely to make a clear distinction between the phenomena since evidence for an interrelation between radiation-induced 
transmissible genomic instability, bystander effects and adaptive responses has become available that be reviewed below.

Radiation-induced genomic instability and bystander effects are closely related regarding mechanisms and effect that persists for many generations. These harmful effects are the same as those occurring in irradiated cells and are presumably a consequence of the deposition of energy in the cell nucleus. These untargeted effects are demonstrated in the descendants cells that have received damaging signals from the irradiated cells (radiation-induced genomic instability) or in cells that are in contact with irradiated cells or receive certain signals from irradiated cells (radiationinduced bystander effects). Radiation-induced genomic instability is characterized by a number of delayed adverse responses such as chromosomal abnormalities, gene mutations and cell death. Similar effects, as well as responses that may be regarded as protective, have been attributed to bystander mechanisms. The process of genomic instability not only induced in progeny of directly irradiated cells but may also be induced in bystander cells.

The initial cross section for radiation damage is increased by the bystander effect, and cells that are affected by the bystander mechanism may remain at an increased risk of genetic change for many generations. There is accumulating evidence that radiation-induced genomic instability may be a consequence of bystander interactions involving intercellular signaling, production of cytokines and free radical generation. Observations of roles for ROS and/or various cytokines in both responses have led to suggestions that the two responses may be interrelated $[21,28]$.

Normal cells have a control mechanism that normally suppresses genomic instability. Cells may normally suppress instability but that radiation may stimulate factors that overcome this suppression and promote an endogenous process. Exposure to ionizing radiation can result in the manifestation of a number of deleterious effects in the multiple generations of irradiated cells that increase the rate of acquisition of alterations in the genome. These dying cells constitute a risk to the cells in culture by releasing a variety of factors such as inflammatory cytokines, nitric oxide-dependent factors, potential nucleases and signaling molecules in addition to the peroxy radicals that can trigger potentially detrimental cellular responses. These reactive intermediates then contribute to a chronic prooxidant environment that cycles over multiple generations, promoting chromosomal recombination and other phenotypes associated with genomic instability. The consequences of genomic instability includes such deleterious endpoints as chromosomal rearrangements, delayed mutation, DNA nucleotide repeat instability, cellular transformation and cell death [21]. It is likely that the process may be a major factor in determining the long term response of populations to low dose of ionizing radiation. The phenomena of radiation-induced genomic instability and bystander effects may reflect interrelated aspects of inflammatory-type responses to radiation-induced stress and injury and contribute to the variety of pathological consequences of radiation exposures.

The term radioadaptive response indicated that the deleterious effects of low dose radiation may be attenuated by a priming low radiation dose that may serve to reduce the effect of subsequent higher dose exposures. Contrary, the harmful effects of low dose radiation may be amplified due to induced bystander responses [21,36] which were not directly traversed by radiation, and which have resulted from some types of communication or signaling between the targeted cells and nearby non-targeted or bystander cells. Bystander effects and adaptive responses are two phenomena that modulate cellular responses to low doses of ionizing radiation that appear to be two conflicting low dose phenomena. Bystander effects on the other hand exaggerate the effects of low doses of radiation by eliciting detrimental effects in non-targeted cells, thus making the target for radiation effects greater than the volume irradiated. Adaptive responses generally indicate that low doses of radiation can reduce damage induced by a second challenging dose. Consequently bystander effects and adaptive responses have the potential to impact on the shape of the dose response profile at low doses of radiation [21,36,50].

Increasing evidence that reactive oxygen and nitrogen species may play roles in correlation between adaptive response and bystander effects [5,6,51]. Zhou et al. [45] have investigated the interaction between a specific bystander effects and adaptive responses. Interestingly, a low adapting dose of radiation decreased mutagenesis mediated by bystander in human hamster $A_{L}$ cells. Thus, the adaptive responses decreases non-targeted bystander mutagenesis. However bystander cells show an increase in sensitivity after a subsequent challenge with X-rays. The similarity and difference in various parameters that can modulate radiation-induced adaptive response and the bystander effect is that both are primarily low-dose phenomena. In general, there are more similarities than differences between the two phenomena. Both the adaptive response and the bystander effect 
have been demonstrated by a range of biological endpoints including cell killing, oncogenic transformation, mutagenesis, chromosomal aberrations, induction of p53 protein, and DNA repair foci. Although bystander effects are not p53 dependent, there are reports that adaptive response, in some studies, is related to p53 function. It should be noted that cancer cells with mutated p53 protein can also demonstrate an adaptive response. Both phenomena involve signals that mediate through either gap junctions or soluble mediators.

Although adaptive response is largely protective in nature and the bystander response, in general, signifies detrimental effects, the two processes share many common characteristics. Although many of the bystander responses reported have been detrimental in nature, there are reported protective effects as well, for example, induction of apoptosis of potentially damaged cells. There are several signaling events that are common to both these low-dose phenomena and suggest that the adaptive response and the bystander effect share a common stress-related signaling lineage. If a cell need not actually be "hit" by radiation to show negative effects, this could affect radiation risk and protection standards. A better understanding of the mechanisms involved in the two processes and how they interact at the cellular, tissue, and organ levels will be important in obtaining a better and more accurate low-dose radiation risk assessment.

\section{CONCLUSION}

The untargeted and delayed effects associated with low dose radiation exposure that still poorly understood are genomic instability, bystander effects and adaptive response. Genomic instability and adaptive response cause a substantial reduction of the risk at low doses, while induction of detrimental bystander effects slightly increase the risk. These non-targeted mechanisms have significant implications for understanding mechanisms of radiation action but the current state of knowledge does not suggest statements about whether these phenomena have implications for assessing radiation risk.

Further studies are now needed to consider how the risk from low levels of radiation exposure might be influenced by these three phenomena. Therefore while some progress has been made in understanding these phenomena, it would be too early to consider revising current risk calculations on the basis of current studies. Scientific understanding of the processes contributing to radiation-induced disease will be used in refinement of judgements on low dose risk.

\section{REFERENCES}

1. E.J. Hall and A.J. Giaccia, Radiobiology for the Radiologist, $7^{\text {th }}$ ed, J.B. Lippincott Company, Philadelphia (2012) 12.

2. Anonymous, Biological Mechanisms of Radiation Actions at Low Doses, United Nations Scientific Committee on the Effects of Atomic Radiation, UNSCEAR, New York (2012) 2.

3. Anonymous, Health Risks from Exposure to Low Levels of Ionizing Radiation: BEIR VII Phase 2, Committee to Assess Health Risks from Exposure to Low Levels of Ionizing Radiation, The National Academies Press, Washington (2006) 11.

4. Anonymous, The 2007 Recommendations of the International Commission on Radiological Protection, ICRP Publication 103, Annals of the ICRP 37 (2-4), International Commission on Radiological Protection, Elsevier (2007).

5. H. Matsumoto, M. Tomita, K. Otsuka et al., J. Radiat. Res. 50 (2009) A67.

6. Y. Zhang, J. Zhou, J. Baldwin et al., Mutat. Res. 671 (2009) 20.

7. K.M. Prism, M. Folkard and B.D. Michael, Oncogene 22 (2003) 7043.

8. J.B. Little, J. Radiol. Prot. 23 (2003) 173.

9. M. Tubianan, J. Dutreix and A. Wambersie, Introduction to Radiobiology, Taylor \& Francis, London (1990) 34.

10. F.E. Mettler, JR and A.C. Upton, Medical Effects of Ionizing Radiation, $3^{\text {rd }}$ ed., Saunders Elsevier, Philadelphia (2008) 9.

11. L.-H. Ding, M. Shingyoji, F. Chen et al., Radiat. Res. 164 (2005) 17.

12. S.A. Amundson, R.A. Lee, C.A. Koch-Paiz et al., Mol. Cancer Res. 1 (2003) 445.

13. S.R. Berglund, D.M. Rocke and J. Dai, Int. J. Radiat. Oncol. Biol. Phys. 70 (2008) 229.

14. P. Fortini and E. Dogliotti, DNA Repair 6 (2007) 398.

15. K.W. Caldecott, DNA Repair 6 (2007) 443.

16. P.A. Jeggo and M. Lobrich, DNA Repair 5 (2006) 1192.

17. E.G. Wright and P.J. Coates, Mutation Research 
597 (2006) 119.

18. S.A. Lorimore, J.A. Chrystal, J.I. Robinson et al., Cancer Res. 68 (2008) 8122.

19. D.I. Portess, G. Bauer, M.A. Hill et al., Cancer Res. 67 (2007) 1246.

20. W.H. McBride, C.S. Chang, J.L. Olson et al., Radiat. Res. 164 (2004) 1.

21. W.F. Morgan, Radiat. Res. 159 (2003) 567.

22. J.Poleszczuk, A. Krzywon, U. Forys et al., Radiat. Res. 183 (2015) 571.

23. K.M. Prise, G. Schettoni, M. Folkard et al., Lancet Oncol. 6 (2005) 520.

24. D. Schulz-Ertner and H. Tsujii, J. Clin. Oncol. 25 (2007) 953.

25. K. Tanaka, A. Kohda, T. Toyokawa et al., Mutat. Res. 657 (2008) 19.

26. R.C. Barber, R.J. Hardwick, M.E. Shanks et al., Mutat. Res 664 (2009) 6.

27. M.H. Barceloss-Hoff and S.V. Costes, Mutat. Res. 597 (2006) 32.

28. S.A. Lorimore, P.J. Coates and E.G. Wright. Oncogene 22 (2003) 7058.

29. H. Yang, N. Asaad and K.D. Held, Oncogene 24 (2005) 2096.

30. N. Hamada, H. Matsumoto, T. Hara et al., Radiat. Res. 48 (2007) 87.

31. W. Han, L. Wu, S. Chen et al., Oncogene 26 (2007) 2330.

32. P.K. Narayanan, K.E.A. LaRue, E.H. Goodwin et al., Radiat. Res. 152 (1999) 57.

33. C. Shao, M. Folkkard and K.M. Prise, Oncogene 27 (2008) 434.

34. H. Zhou, V.N. Ivanov, Y.-C. Lien et al., Cancer Res. 68 (2008) 2233.
35. P.J. Coates, J.K. Rundle, S.A. Lorimore et al., Cancer Res. 68 (2008) 450.

36. O.V. Belyakov, M. Folkard, C. Mothersill et al., Br. J. Cancer 88 (2003) 767.

37. O.A. Scdelnikova, A. Nakamura, O. Kovalchuck et al., Cancer Res. 67 (2007) 4295.

38. A.I. Kassis, Hum. Exp. Toxicol. 23 (2004) 71.

39. A. Van der Meeren, P. Monti, M. Vandamme et al., Radiat. Res. 163 (2005) 144.

40. G. Olivieri, Y. Bodycote and S. Wolff, Science 223 (1984) 594.

41. M.M. Vilenchik and A.G. Knudson, Proc. Natl. Sci. 100 (2003) 12871.

42. J.F. Ward, Prog. Nucleic Acid Res. Mol. Biol. 35 (1988) 95.

43. L.E. Feinendegen, Hum. Exp. Toxicol. 18 (1999) 426.

44. H. Gourabi and H. Mozdarani, Mutagenesis 13 (1998) 475.

45. H. Zhou, G. Rabders-Pehrson, C.R. Geard et al., Radiat. Res. 160 (2003) 512A.

46. L. Redpath and R.J. Antoniono, Radiat. Res. 149 (1998) 517.

47. S.J. Hyun, M.Y. Yoon, T.H. Kim et al., Anticancer Res. 17 (1997) 225.

48. K. Parmar, P. Mauch, J.-A. Vergilio, Proc. Natl. Sci. 104 (2007) 5431.

49. M.A. Coleman, E. Yin, L.E. Peterson et al., Radiat. Res. 164 (2005) 369.

50. C. Mothersill and C. Seymour, Mutat. Res. 568 (2004) 121.

51. M.A. Kadhim, S.R. Moore and E.H. Goodwin, Mutat. Res. 568 (2004) 21. 